\title{
Editorials
}

\section{Choosing antithrombotics for atrial fibrillation in primary care}

Atrial fibrillation (AF) is the most common cardiac arrhythmia in the UK affecting 1.3 million people. ${ }^{1} \mathrm{AF}$ is particularly common in older people, with one in ten over the age of 75 years affected. ${ }^{2}$ The prevalence of $A F$ is projected to increase with improved survival of patients from chronic cardiac disorders that predispose to AF, such as coronary heart disease. AF increases the risk of stroke fivefold due to thromboembolism and cause $15 \%$ of all strokes. ${ }^{3}$

\section{STRATIFYING PATIENTS WITH ATRIAL FIBRILLATION FOR STROKE PREVENTION}

Anticoagulation therapy reduces the risk of ischaemic stroke in patients with AF. When deciding on treatment, the National Institute for Health and Care Excellence (NICE) AF management guidelines recommend undertaking a risk benefit analysis using the $\mathrm{CHA}_{2} \mathrm{DS}_{2}$-VASc score to estimate an individual's risk of stroke and the HAS-BLED score to estimate the risk of bleeding. ${ }^{4}$ For most people, the benefit of anticoagulation outweighs the risk of bleeding and so a high HAS-BLED score should not be used to withhold stroke prophylaxis but rather prompt more careful and frequent monitoring.

\section{ANTITHROMBOTIC OPTIONS}

In recent years, the recommended type of antithrombotic as a first line therapy has changed in order to maximise stroke risk reduction while minimising the risk of bleeding. In a meta-analysis of six trials, oral anticoagulants, such as the vitamin $\mathrm{K}$ antagonist (VKA) warfarin, reduced the risk of ischaemic stroke by $64 \%$ and all-cause mortality by $26 \%$ compared to placebo or no treatment in individuals with non-valvular $A F^{5}{ }^{5}$ In 2011, non-VKA oral anticoagulants (NOACs), such as dabigatran, apixaban, and rivaroxaban were licensed in the UK as alternatives to warfarin and recommended in the 2014 NICE guidelines. ${ }^{4}$ This recommendation was based on a meta-analysis of four trials demonstrating that NOACs confer additional reductions in risk of stroke or systemic thromboembolism and all-cause mortality by $19 \%$ and $10 \%$ respectively, relative to warfarin, with a similar risk of major bleeding but an increased risk of gastrointestinal bleeding. ${ }^{6}$

Despite NOACs being shown to be generally non-inferior or superior to VKA in terms of benefit and safety outcomes, VKA therapy still has a role to play in circumstances when NOACs are contraindicated; for example, in patients with renal failure or 'valvular' AF. The use of international normalised ratio levels to monitor warfarin's anticoagulant effect and adjust dosing to maximise benefit and minimise risk is also a perceived advantage. Furthermore, in order to optimise the quality of VKA therapy, NICE guidelines recommend the use of point-of-care coagulometers for patients to self-monitor and self-manage anticoagulation. ${ }^{4}$

In contrast, NOACs have a rapid onset and offset of action providing for more predictable pharmacokinetics and therefore patients who use NOACs do not need regular blood tests for anticoagulation monitoring. NOACs also have fewer drug and dietary interactions. Even without continuous monitoring of treatment effect, periodic assessments, including blood tests, are required with NOAC use to re-evaluate risk factors for bleeding and potential contraindications, such as anaemia, thrombocytopenia, renal failure, or liver disease. Although patient persistence with NOACs is significantly higher than with VKA, ongoing contact with clinicians will also provide further opportunity to support patients to optimise adherence and continue NOACs as interruption to therapy is associated with increased risk of stroke. ${ }^{7.8}$

In the absence of any head-to-head trials, guidelines do not currently recommend any particular NOAC over the others when treating patients with $A F$, but emerging protocols instead offer pointers to treatment choice on an individualised case-by-case approach. The various considerations, ranging from age and weight to renal function and dosing regimen, makes choosing a particular NOAC in practice a complex process and reflects their safety profiles in different study populations in each of the NOAC trials. ${ }^{6}$

Observational studies have estimated the effectiveness and safety of individual antithrombotics outside clinical trial settings. In a large UK prospective open cohort study comparing the three most common types of NOACs with warfarin, apixaban was found to be the safest drug with a decreased risk of major bleeding ladjusted hazard ratio [HR] $0.66,95 \%$ confidence interval $[\mathrm{Cl}]=0.54$ to 0.79 ) and intracranial bleeding ladjusted $\mathrm{HR} 0.40, \mathrm{Cl}=0.25$ to 0.64); however, compared to warfarin, an increased risk of allcause mortality was observed for rivaroxaban ladjusted HR 1.19, $\mathrm{Cl}=1.09$ to 1.29) and low dose apixaban ladjusted HR 1.27, $\mathrm{Cl}=1.12$ to 1.45$).{ }^{10}$
Observational data are generally limited by residual confounding and therefore argued insufficient to guide selection of individual anticoagulants but instead used to reaffirm concordance with clinical trial findings. In a meta-analysis of observational studies, the results were generally consistent with those of the trials and showed that individual NOACs were similar or better than warfarin in terms of the rates of prevention of thromboembolic events and major bleeding in patients with non-valvular AF."1 A study providing a rank order for use of OACs for stroke prevention in patients with AF also noted apixaban was the most cost effective. ${ }^{12}$

\section{VALVULAR VERSUS NON-VALVULAR ATRIAL FIBRILLATION}

Antithrombotic choice in AF is linked to whether the origin of AF is related to valvular or non-valvular heart disease. Previous NOAC versus warfarin trials have focused on patients with non-valvular AF on the account of the different pathogenesis, risk of thromboembolism, and treatment needs due to valvular AF. ${ }^{6}$ While non-valvular AF referred to AF in the absence of a mechanical prosthetic heart valve or moderate to severe mitral stenosis (usually of rheumatic origin), patients with other valvular heart disease were included in the NOAC trials. ${ }^{6}$ This created some uncertainty among doctors as to the precise definition of 'valvular' AF and 'nonvalvular' AF leading to therapeutic confusion.

The European Heart Rhythm Association has reclassified 'non-valvular' AF, preferring instead to categorise it as AF with valvular heart disease', with two further subtypes influencing choice of oral anticoagulant thromboprophylaxis. ${ }^{13}$ Evaluated Heartvalves, Rheumatic or Artificial (EHRA) type 1 refers to AF with moderate-severe mitral stenosis of rheumatic origin and mechanical prosthetic valve needing VKA. Whereas, EHRA type 2 refers to patients with AF with all other native valvular stenoses and insufficiencies as well as mitral valve repair, bioprosthetic valve replacements, and transaortic valve intervention when a VKA or NOAC is indicated. Based on this classification, patients with EHRA type 2 valvular heart disease were variously included in the NOAC trials. ${ }^{13}$

\section{ATRIAL FIBRILLATION AND CORONARY HEART DISEASE}

Clinicians in primary care face the challenge of managing patients with both $A F$ and 
stable coronary heart disease ( $\mathrm{CHD}$ ) when there is indication for using anticoagulants and antiplatelets in the prevention of stroke and myocardial infarction respectively. Approximately $20 \%-40 \%$ of patients with AF have concomitant $\mathrm{CHD}^{14}$ In the UK, $\mathrm{CHD}$ remains the most common cause of premature death ( $<75$ years of age), responsible for around 15\% of premature male deaths and $8 \%$ of premature female deaths. ${ }^{1}$ The findings of the meta-analysis of 16 secondary prevention trials comparing the long-term benefit of aspirin versus control in vascular disease has helped establish aspirin therapy in guidelines for the management of $\mathrm{CHD}$ (for example, myocardial infarction) and its recurrence. ${ }^{15}$

The European guideline advocates patients with $\mathrm{AF}$ and stable $\mathrm{CHD}$ can be managed with lifelong OAC without aspirin as that would otherwise increase the risk of bleeding: ${ }^{16}$ however, guidelines on co-prescribing multiple antithrombotic medications in patients with $\mathrm{AF}$ and $\mathrm{CHD}$ are based on limited observational data and expert opinion. 4,16

In a Danish cohort study, the addition of antiplatelet therapy to warfarin in patients with $\mathrm{AF}$ and stable $\mathrm{CHD}$ (defined as, 12 months from an acute coronary event: myocardial infarction or percutaneous coronary intervention) was not associated with a reduction in the risk of recurrent coronary events or thromboembolism, whereas the risk of bleeding was increased significantly..$^{17}$ A recent randomised trial in Japan comparing OAC alone to combined $O A C$ and a single antiplatelet in patients with $\mathrm{AF}$ and stable $\mathrm{CHD}$ was stopped early because of increased mortality in the combination therapy group. ${ }^{18}$ However, compared with Western populations, in Japan there is a higher prevalence of hypertension, risk of stroke and bleeding with antiplatelets. ${ }^{19}$

\section{CONCLUSION}

Stroke prevention in patients with AF in primary care requires careful consideration of the benefit and bleeding risk of multiple different antithrombotic options. For patients with AF with EHRA type 1 valvular heart disease, a VKA may be indicated. In those with EHRA type 2 valvular heart disease, NOACs are widely considered to be the antithrombotic of choice.

In patients with $\mathrm{AF}$ and $\mathrm{CHD}$, evidence for when to treat and with which antithrombotic agents is less clear. To inform treatment decisions in this group of patients, further work is needed to better understand the risk of bleeding according to individual antithrombotic type, dose, and intended duration, particularly since those affected will tend to be older, with comorbidities and polypharmacy, and with an increased risk of bleeding that can occur suddenly and be lifethreatening.

\section{Mohammed Mustafa,}

GP Principal, Grange Medical Practice, Cardiff, UK.

\section{James P Sheppard,}

University Research Lecturer, Nuffield Department of Primary Care Health Sciences, University of Oxford, Oxford; Senior Fellow, National Institute for Health Research, Oxford Biomedical Research Centre, UK.

\section{Provenance}

Freely submitted; externally peer reviewed.

\section{REFERENCES}

1. British Heart Foundation. UK factsheet December 2019. 2019. https://uww.bhf.org. uk/-/media/files/research/heart-statistics/ bhf-cvd-statistics-uk-factsheet.pdf?la=en laccessed 7 Jan 2020).

2. Hobbs FDR, Fitzmaurice DA, Mant J, et al. A randomised controlled trial and costeffectiveness study of systematic screening (targeted and total population screening) versus routine practice for the detection of atrial fibrillation in people aged 65 years and over. The SAFE study. Health Technol Assess 2005; 9(40): iii-iv, ix-X, 1-74.

3. Wolf PA, Abbott RD, Kannel WB. Atrial fibrillation as an independent risk factor for stroke: the Framingham study. Stroke 1991; 22(8): 983-988.

4. National Institute for Health and Care Excellence. Atrial fibrillation: management. CG180. 2014. https://www.nice.org.uk/ guidance/cg180 (accessed 30 Dec 2019).

5. Hart RG, Pearce LA, Aguilar MI. Metaanalysis: antithrombotic therapy to prevent stroke in patients who have nonvalvular atrial fibrillation. Ann Intern Med 2007; 146(12): 857-867.

6. Ruff CT, Giugliano RP, Braunwald E, et al. Comparison of the efficacy and safety of new oral anticoagulants with warfarin in patients with atrial fibrillation: a meta-analysis of randomised trials. Lancet 2014; 383(9921): 955-962.

7. Martinez C, Katholing A, Wallenhorst C, Freedman SB. Therapy persistence in newly diagnosed non-valvular atrial fibrillation treated with warfarin or NOAC. A cohort study. Thromb Haemost 2016; 115(1): 31-39

8. Sørensen R, Nielson BJ, Pallisgaard JL, et al. Adherence with oral anticoagulation in non-valvular atrial fibrillation: a comparison of vitamin $\mathrm{K}$ antagonists and non-vitamin Kantagonists. Eur Heart J Cardiovasc Pharmacother 2017; 3(3): 151-156.

9. National Institute for Health and Care Excellence, Boehringer Ingelheim Ltd, Prescribing Decision Support Ltd. Decision support: anticoagulation therapy for the prevention of stroke and systemic embolism in atrial fibrillation. 2017. https://mww.anticoagulation-dst.co.uk (accessed 30 Dec 2019).

\section{ADDRESS FOR CORRESPONDENCE}

Mohammed Mustafa

Grange Medical Practice, 25 Bishop Street, Cardiff CF11 6PG, UK.

\section{Email: mustafamhRacardiff.ac.uk}

\section{Competing interests}

James P Sheppard is a National Institute for Health Research (NIHR) Oxford Biomedical Research Centre Senior Fellow and receives funding from the Wellcome Trust/Royal Society via a Sir Henry Dale Fellowship (ref: 211182/Z/18/Z); the NIHR School for Primary Care Research; and the NIHR Collaboration for Leadership in Applied Health Research and Care Oxford at Oxford Health NHS Foundation Trust.

\section{DOI: https://doi.org/10.3399/bjgp20X708317}

10. Vinogradova Y, Coupland C, Hill T, HippisleyCox J. Risks and benefits of direct oral anticoagulants versus warfarin in a real world setting: cohort study in primary care. BMJ 2018; 362: k2505.

11. Ntaios G, Papavasileiou V, Makaritsis K, et al. Real-world setting comparison of nonvitamin-K antagonist oral anticoagulants versus vitamin- $\mathrm{K}$ antagonists for stroke prevention in atrial fibrillation. a systematic review and metaanalysis. Stroke 2017; 48(9): 2494-2503.

12. López-López JA, Sterne JAC, Thom HHZ, et al. Oral anticoagulants for prevention of stroke in atrial fibrillation: systematic review, network meta-analysis, and cost effectiveness analysis BMJ 2017; 359: j5058.

13. Steffel J, Verhamme P, Potpara TS, et al. The 2018 European Heart Rhythm Association Practical Guide on the use of non-vitamin K antagonist oral anticoagulants in patients with atrial fibrillation. Eur Heart J 2018; 39(16): 1330-1393.

14. Hansen ML, Sørensen R, Clausen MT, et al. Risk of bleeding with single, dual, or triple therapy with warfarin, aspirin, and clopidogrel in patients with atrial fibrillation. Arch Intern Med 2010; 170(16): 1433-1441

15. Antithrombotic Trialists' (ATT) Collaboration Baigent C, Blackwell L, et al. Aspirin in the primary and secondary prevention of vascular disease: collaborative meta-analysis of individual participant data from randomised trials. Lancet 2009; 373(9678): 1849-1860.

16. Kirchhof P, Benussi S, Kotecha D, et al. 2016 ESC Guidelines for the management of atrial fibrillation developed in collaboration with EACTS. Eur Heart J 2016; 37(38): 2893-2962.

17. Lamberts M, Gislason GH, Olesen JB, et al. Oral anticoagulation and antiplatelets in atrial fibrillation patients after myocardial infarction and coronary intervention. J Am Coll Cardiol 2013; 62(11): 981-989

18. Yasuda S, Kaikita K, Akao M, et al. Antithrombotic therapy for atrial fibrillation with stable coronary artery disease. N Engl J Med 2019: 381(12): 1103-1113.

19. Yun JE, Kim YJ, Park JJ, et al. Safety and effectiveness of contemporary P2Y12 inhibitors in an East Asian population with acute coronary syndrome: a nationwide population-based cohort study. J Am Heart Assoc 2019; 8(14): e012078. 